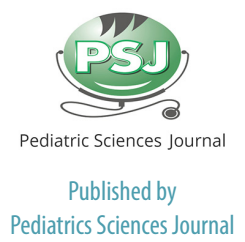

Pediatrics Sciences Journal

\section{Stroke in pediatric: a review}

\author{
Nugroho Danu ${ }^{1 *}$, Melinda $^{1}$, Astarini Hidayah ${ }^{2}$
}

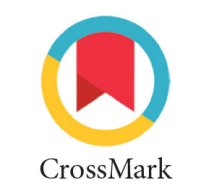

CrossMark
${ }^{1}$ Neuropediatric Division, Pediatric Department, Faculty of Medicine, Universitas Brawijaya, Malang, East Java, Indonesia

2Pediatric Department, Faculty of Medicine, Universitas Brawijaya, Malang, East Java, Indonesia

*Corresponding to:

Nugroho Danu; Neuropediatric Division,

Pediatric Department, Faculty of

Medicine, Universitas Brawijaya, Malang,

East Java, Indonesia;

danu.dr@ub.ac.id

Received: 2021-03-16

Accepted: 2021-05-20

Published: 2021-06-01

\section{ABSTRACT}

Stroke in children was defined as the clinical and radiological manifestation of stroke, with radiological evidence of cerebral infarction or hemorrhage. Pediatric stroke can be divided into ischemic and hemorrhagic stroke. The etiology is very different, and diagnostic procedures are often more in-depth to look for etiology. Stroke is classically characterized by acute focal neurologic deficits such as hemiplegia, speech, or gait disturbances; presentation is highly dependent on the child's age and may be non-specific. When acute focal neurologic deficits are found in childhood, one should think about stroke until proven otherwise. There is no standard therapy based on a systematic review of a randomized clinical trial. Therapeutic recommendations are the consensus of experts, extrapolated from the recommendations for stroke therapy for the adult population. Understanding stroke in children is complicated because it has broad differential diagnosis, and complications that occur. Therefore, early recognition of childhood stroke by pediatricians is very important with the implementation of targeted and neuroprotective therapies.

Keywords: Stroke, Children, Diagnosis, Treatment, Prognosis.

Cite This Article: Danu, N., Melinda., Hidayah, A. 2021. Stroke in pediatric: a review. Pediatrics Sciences Journal 2(1): 20-28.

\section{INTRODUCTION}

Stroke is one of the main causes of disability and can lead to death. Stroke in children was defined as the clinical and radiological manifestation of stroke, with radiological evidence of cerebral infarction or hemorrhage. ${ }^{1}$ Stroke in children can be categorized into (1) neonatal stroke which includes prenatal, perinatal, and neonatal stroke (up to the first month of life), and (2) non-neonatal stroke or childhood stroke (over 1 month of age). ${ }^{1}$

Like adult stroke, pediatric stroke can be divided into ischemic and hemorrhagic stroke. Ischemic stroke is defined as focal damage to the blood vessels of brain tissue due to loss of blood flow or oxygenation. ${ }^{2}$ Hemorrhagic stroke is a spontaneous bleeding in the brain parenchyma (intraparenchymal hemorrhage) and spontaneous subarachnoid hemorrhage (nontraumatic) directly adjacent to the brain's surface. ${ }^{3}$ Bleeding causes focal brain damage through local mass effects and ischemia in surrounding tissues. Stroke in children occurs from 2.3 to 13 per 100,000 children per year. Strokes can occur in apparently healthy infants, children, and adolescents. ${ }^{2,3}$ Manifestations of childhood stroke symptoms are often non-specific, stroke should be suspected in acute neurologic clinical changes. In the neonatal age, seizures are the most common symptom. Research by Zimmer JA et al., found that seizures were the largest proportion (45\%) of stroke symptoms in children $<1$ year. ${ }^{4}$ In older children, symptoms of focal neurologic deficits such as hemiparesis, loss of consciousness, and cranial nerve lesions are more common. ${ }^{2-4}$

In contrast to the adult population, randomized clinical trials of stroke therapy in children are very limited. Management is more general in nature to stabilize hemodynamic conditions, oxygen supplementation, and temperature control. $^{5}$ AHA recommendations (American Heart Association) recommend the use of low molecular weight heparin or acetylsalicylic acid $(1 \mathrm{mg} / \mathrm{kg} /$ day $)$ until the etiology of ischemic stroke in children is established. In hemorrhagic stroke, therapy is carried out to reduce the mass effect and to prevent or reduce the herniation syndrome. ${ }^{5}$

Stroke in children is quite rare, but it is important to discuss because it has risk factors, different clinical features from stroke in adults, wide differential diagnosis, and complications that occur. Early recognition of childhood stroke by pediatricians is very important with the implementation of targeted and neuroprotective therapies. ${ }^{3}$ The high rate of morbidity and mortality in pediatric stroke makes this paper important so that it can determine the management strategy with an integrated multidisciplinary approach.

Based on those mentioned above, this article will describe the definition, classification, etiology and risk factors, how to make a diagnosis, the effectiveness of therapy and the prognosis of stroke in children.

\section{ANATOMY OF THE CIRCULATORY BLOOD VESSELS OF THE BRAIN}

The four major arteries supplying blood to the brain are the two internal carotid arteries and the two vertebral arteries (which fuse with the basilar artery to 
form the vertebrobasilar system). Arterial blood to the brain comes from the aortic arch. In general, the cerebral arteries are penetrating or conducting. ${ }^{6}$ The conducting arteries (carotid, middle and anterior cerebral, vertebral, basilar, and posterior cerebral) and their branches form an extensive network on the surface of the brain. In general, the carotid arteries and their branches supply the largest part of the cerebral hemispheres, and the vertebral arteries supply the base of the brain and cerebellum. The penetrating arteries are the vessels that carry food and originate from the conducting arteries. These vessels enter the brain with and supply blood to structures located below the cortex (thalamus, hypothalamus, internal capsule, and basal ganglia). ${ }^{6}$

Collateral circulation can be formed slowly when there is a decrease in normal blood flow to a part. Most of the cerebral collateral circulation between the great arteries is via the Circulus of Willis. The effect of this collateral circulation is to ensure the distribution of blood flow to the brain. These collaterals only function when other routes are interrupted. The gray matter of the brain has a much higher metabolic rate than that of the white matter, so the number of capillaries and blood flow is also four times greater.

Peripheral lesions involving the cortex and white matter are characterized by infarction due to occlusion of the great arteries. Most arterial ischemic strokes involve the middle cerebral artery. ${ }^{8}$ There is a pattern of vascularization based on the occlusion of the artery, namely: (1) proximal M1 occlusion (all middle cerebral artery infarction), (2) M1 distal occlusion (basal ganglia), (3) Anterior or posterior M2 occlusion (frontal or parietal/temporal), (4) lenticulosstriata (basal ganglia and deep white matter). Specific vascular patterns can help predict the etiology of ischemic stroke which is commonly associated with arteriopathies. ${ }^{8}$

\section{DEFINITION AND CLASSIFICATION OF STROKE IN CHILDREN}

The standard definition of stroke in adults is acute-onset neurological signs or symptoms caused by focal brain infarction or hemorrhage and this definition applies to pediatrics as reflected in the National Institutes of Health (NIH). Pediatric stroke can also be classified according to the underlying disease, namely ischemic and hemorrhagic. ${ }^{9}$ Classified ischemic stroke, namely Arterial Ischemic Stroke (AIS) and venous infarction by Cerebral Synovenous Thrombosis (CSVT) or cortical vein thrombosis. In CSVT, venous sinus obstruction may or may not be accompanied by bleeding. In infants and older children, some literature uses the term silent stroke when asymptomatic infarction is found on neuroimaging. Although this is wrong because the

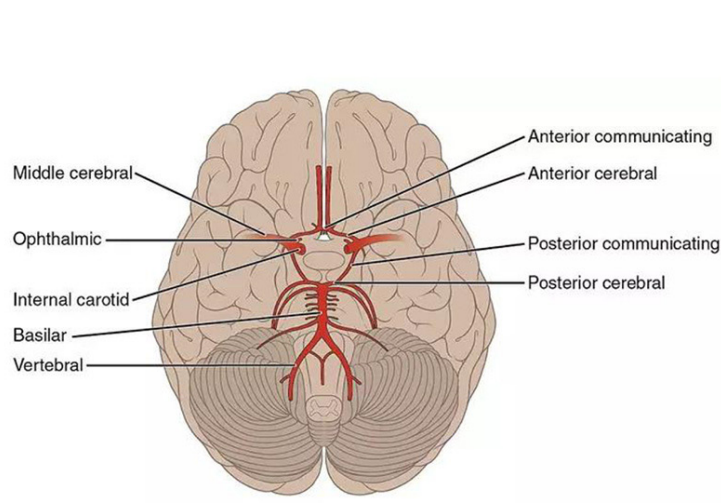

Figure 1. Cycle of Willis. The circle of Willis is an arterial polygon (heptagon) formed by the internal carotid system and vertebral anastomoses around the optic chiasm and pituitary infundibulum in the suprasellar cistern. This communication pathway allows distribution of blood flow between the two sides of the brain, and allows anastomotic circulation, if part of one side of the circulation is obstructed. ${ }^{6}$ definition of stroke includes clinical conditions. However, silent infarctions may not be completely asymptomatic; as in adults, the disorder is likely to cause vascular cognitive impairment. ${ }^{9}$

Ischemic stroke occurs in approximately 1.0 to 2.0 in 100,000 children each year in Western countries. Incidence varies by age and sex; highest in infants and children $<5$ years and higher in boys than girls. Black and Asian children have a higher incidence than white children. Most of the increased risk of ischemic stroke in black children is due to Sickle Cell Disease (SCD), which increases stroke risk $>200$ fold. ${ }^{10}$ Hemorrhagic strokes in children often occur in the form of Intracerebral Hemorrhage (ICH), Intraventricular Hemorrhage (IVH), or Subarachnoid Hemorrhage (SAH). Hemorrhagic strokes account for half of strokes in children, with a rate of 1 to 1.7 in 100,000 per year. ${ }^{10}$

Most arterial ischemic strokes involve the middle cerebral artery. There is a pattern of vascularization based on the occlusion of the artery, namely: (1) proximal M1 occlusion (all middle cerebral artery infarction), (2) M1 distal occlusion (basal ganglia), (3) Anterior or posterior M2 occlusion (frontal or parietal/temporal), (4) lenticulosstriata (basal ganglia and deep white matter). Specific vascular patterns can help predict the etiology of ischemic stroke which is commonly associated with arteriopathies. ${ }^{8}$

\section{RISK FACTOR AND ETIOLOGY OF STROKE IN CHILDREN}

Childhood stroke is known as a disease with multiple risks, as displayed in Table 1. Arterial ischemic stroke in children often has no proven risk factor, some risk factors are presumptive risk factors. ${ }^{11}$ Research shows that cardiac anatomical anomalies in pediatric stroke cases are 2-3 times that of the general population. Congenital heart disease is responsible for $1 / 3$ of ischemic stroke cases in children. Among congenital heart disease, cyanotic congenital heart disease with right to left shunt has the highest risk. This is due to a more severe degree of hypoxaemia, cyanosis, and secondary polycythemia. In patients undergoing surgery, the perioperative period is particularly at risk for stroke; this is because the greater the 
risk of cerebral hypoperfusion by a low cardiac index and cardiac arrhythmias. Another factor that should be considered as a risk factor for stroke is bacterial endocarditis with septic embolism. ${ }^{12}$

Sickle cell disease is one of the most common causes of stroke in children. The incidence of sickle cell disease is $0.13 \%$ in infants less than 1 year of age, and increases to $1 \%$ in children $2-5$ years. The incidence of stroke in patients with sickle cell disease is 280 times that of the general population. Stroke in sickle cell disease occurs due to vasculopathy in the distal part of the internal carotid artery, and the proximal part of the middle cerebral artery and anterior cerebral artery. ${ }^{13}$

One of the other causes of stroke in children is Moyamoya disease (Japanese for smog), referring to the arteriography depiction of collateral branches of arteries. Moyamoya's disease is characterized by chronic and progressive stenosis of the distal part of the internal carotid artery, and proximal to the middle, anterior, and posterior cerebral arteries. Prothrombic conditions are present in $>50 \%$ of cases of acute ischemic stroke in children; the most common is protein $\mathrm{C}$, $S$, or antithrombin III deficiency. Other factors that are also commonly found in cases of ischemic stroke in children are hyperhomocysteinemia and high levels of lipoprotein A. ${ }^{13}$

There were several etiologies of hemorrhagic stroke in children as presented in Table 2. Intracerebral hemorrhage $(\mathrm{ICH})$ refers to bleeding

Table 1. Common Risk Factors for Pediatric Acute Ischemic Stroke ${ }^{13}$

\begin{tabular}{|c|c|}
\hline Category & Risk Factors \\
\hline \multirow[t]{15}{*}{ Arteriopathy } & Inflammation/parainfection \\
\hline & Childhood primary angina of CNS (pPACNS) \\
\hline & Transient Cerebral Arteriopathy \\
\hline & Focal Cerebral Arteriopathy \\
\hline & Post Varicella Angiopathy \\
\hline & Infection \\
\hline & Bacterial meningitis and tuberculosis \\
\hline & HIV \\
\hline & dissection \\
\hline & Internal Carotid Artery \\
\hline & Vertebral Artery \\
\hline & Intracranial Artery \\
\hline & Moyamoya Disease (Idiopathic) \\
\hline & Moyamoya syndrome \\
\hline & Neurofibromatosis-1, Trisomy 21 \\
\hline \multirow[t]{9}{*}{ Cardiac } & Complex congenital heart disease \\
\hline & Cardiac surgery (eg Fontan) \\
\hline & Cardiac catheterization (eg BAS) \\
\hline & Other Cardiac Conditions \\
\hline & Bacterial endocarditis \\
\hline & Atrial Septal Aneurysm \\
\hline & Atrial Septal Defect \\
\hline & Patent Oval Foramen \\
\hline & Venous Thrombosis R-L shunt \\
\hline \multirow[t]{6}{*}{ Prothrombotic } & Leiden factor $\mathrm{V}$ \\
\hline & Gene Prothrombin 20210A \\
\hline & Increased Lipoprotein \\
\hline & Protein C Deficiency \\
\hline & Lupus Anticoagulants \\
\hline & Anticardiolipin antibodies \\
\hline \multirow[t]{2}{*}{ Hematology } & Sickle Cell Disease \\
\hline & Severe Deficiency Anemia \\
\hline Drug & Chemotherapy (L-asparaginase) \\
\hline \multirow[t]{2}{*}{ Others } & Migraine \\
\hline & $\begin{array}{l}\text { Inborn Errors of Metabolism: Fabry disease, homocystinuria, } \\
\text { mitochondrial }\end{array}$ \\
\hline
\end{tabular}

within the brain parenchyma and/ or ventricles, whereas subarachnoid hemorrhage (SAH) refers to bleeding in the subarachnoid space. About half of strokes in children are ICH and SAH. Data from the California Database, the incidence of $\mathrm{ICH}$ and $\mathrm{SAH}$ in pediatric patients is $0.8 / 100,000 /$ year and $0.4 / 100,000 /$ year, respectively. ${ }^{14}$ Hemorrhagic stroke focuses on nontraumatic events or spontaneous ICH and SAH caused by ruptured vascular malformations. Most spontaneous childhood ICH and SAH are due to secondary causes such as ruptured vascular malformations, tumors, brain infections, or haematological abnormalities. ${ }^{15}$ Even after a complete examination, $9 \%-23 \%$ of childhood ICH/ $\mathrm{SAH}$ is idiopathic. Vascular malformations are responsible for approximately $17.5 \%$ - $73.5 \%$ of pediatric ICH; arteriovenous malformations are more common than cavernous malformations. ${ }^{13}$

\section{PATHOPHYSIOLOGY OF STROKE IN CHILDREN}

\section{Acute Ischemic Stroke}

The mechanism that explains the occurrence of arterial ischemic stroke is thrombotic occlusion. The difference

Table 2. Etiology of Hemorrhagic Stroke in Children ${ }^{16}$

\section{Etiology}

Vascular malformations

Arterial Malformation (AVM)

Ateriovenous Fistula

Cavernous hemangioma

Aneurysm

Capillary telangiectasia

Venous Malformation

Vasculopathy

Coagulation Disorders

Thrombocytopenia

hemophilia

Liver Disorder

Vitamin K. Deficiency

Anticoagulant Therapy

Medical treatment

Brain tumor

Trauma/Dissection

Unknown 
between thrombus and embolism as the cause of an ischemic stroke is still not clear so that currently both are classified into the same group, namely ischemic stroke. Thrombus can travel locally in the artery or spread to become a source of embolism. Thrombosis is a product of coagulation (fibrin formation) and the platelet hemostasis system. The balance of this system varies depending on blood flow, stress factors, endothelial integrity, anticoagulant levels, and other factors. In static conditions of blood flow, the coagulation system is more involved, but the balance depends on the underlying disease. Exposure to inflammation of the arterial walls such as vasculitis or damage to the endothelium by collagen and tissue factors such as platelet dissection activity and fibrin formation. Changes in the maturation of coagulation and platelets in children also influence this occurrence. ${ }^{13}$

The pathophysiology that explains the mechanism of infarction in arterial ischemic stroke is the severity of cerebral tissue damage. It is related to multiple factors: (1) duration and presence of ischemia and reperfusion time, (2) blood supply ability of the collateral arteries, (3) component function and volume of the affected brain structures, (4) maturation status of the brain, (5) the disease process at the time of stroke and the metabolic demands of ischemic brain tissue. ${ }^{13}$

In ischemic stroke, acute occlusion of an intracranial blood vessel results in decreased blood flow to the vascularized parts of the brain. The importance of reducing blood flow is the functioning of the collateral arteries and this depends on the anatomy of the individual vessel, the site of the occlusion, and sometimes the systemic blood pressure. ${ }^{17}$ Decreased blood flow to the brain to zero causes brain tissue death in 4-10 minutes; value in one hour; and values $<20 \mathrm{ml} / 100 \mathrm{~g}$ brain tissue per minute can cause ischemia without infarction unless it occurs over several hours or days. If blood flow returns to normal before enough cells die, the patient will only experience temporary symptoms and this clinical syndrome is known as Transient Ischemic Attack (TIA). ${ }^{17}$

Cerebral infarction mainly occurs through two pathways, namely: (1) the necrosis pathway where there is rapid breakdown of the cellular cytoskeleton due to the lack of energy in the cells; and (2) the apoptotic pathway by which cells are programmed to die. Ischemia causes necrosis by depriving neurons of glucose and oxygen, which causes mitochondria to fail to produce Adenosine Triphosphate (ATP). Without ATP, the ion pump in the cell membrane stops functioning and causes neuronal depolarization, and causes intracellular calcium to increase. ${ }^{17}$ Cellular depolarization will cause the release of glutamate from the synaptic terminal where excess extracellular glutamate will cause neurotoxicity by activating glutamate receptors which will increase neuronal calcium influx. Lipid membrane degradation and mitochondrial dysfunction will produce free radicals. Free radicals will damage membranes and sometimes damage other vital functions of cells. Milder ischemia is seen in penumbral ischemia, where the process of apoptosis causes the death of brain cells days or weeks later. ${ }^{17}$

Neuronal dysfunction in ischemia involves irreversible infarction with increased duration or degree of ischemia or increased metabolic activity. At TIA no permanent parenchymal lesions were found on MRI. In AIS, there is decreased perfusion in the central zone resulting in an unstable penumbral zone where collateral perfusion attempts to maintain neuronal viability. In the penumbra area, factors that can increase the difference between metabolism and oxygen and glucose supply increase tissue injury and cell death. Changes that follow are the presence of seizures, changes in temperature, blood pressure and serum glucose. This is the main catch point of neuroprotective therapy in stroke as a savior of the penumbra tissue to repair brain tissue that can still function. ${ }^{13}$

Vascular anomalies account for about $45 \%$ of nontraumatic hemorrhagic strokes in children. Arteriovenous malformation (AVM) in which abnormal arteries are connected directly to venous drainage without capillary involvement. The child may have multiple AVMs, especially in genetic syndromes such as hereditary hemorrhagic telangiectasia. Symptomatic aneurysms are relatively rare in children but are associated with conditions such as coarctation of the aorta, polycystic kidney disease, sickle cell disease, Ehlers-Danlos type IV, and fibromuscular dysplasia. The cause of aneurysm rupture is related to the dependence of the aneurysm wall depending on the diameter and the pressure difference inside and outside the aneurysm. After rupture, blood seeps into the subarachnoid space and spreads throughout the brain and spinal cord along with the cerebrospinal fluid. This blood in addition to causing increased intracranial pressure, can also injure brain tissue directly because of the high pressure when it first bursts, and irritates the lining of the brain. ${ }^{18}$

\section{CLINICAL FEATURES OF STROKE IN CHILDREN}

Stroke is classically characterized by acute focal neurologic deficits such as hemiplegia, speech or gait disturbances, presentation is highly dependent on the age of the child and may be non-specific. When an acute focal neurologic deficit is found in childhood, what should be considered is a stroke until proven otherwise. Clinical features in toddlers and infants tend to be the least specific, such as irritability, drowsiness, lethargy, feeding difficulties, apnea and hypotonia. Approximately $30-50 \%$ of children with an acute neurologic presentation will have a non-vascular diagnosis. ${ }^{19}$

Signs and symptoms of acute stroke in children are similar to those of adults. The most common symptoms include hemiparesis and hemifacial weakness in $67 \%-90 \%$, speech or language impairment in $20 \%$ - 50\%, visual impairment in $10 \%$ $15 \%$, and ataxia in $8 \%-10 \%$. In children also accompanied by non-specific symptoms such as headache in $20 \%-50 \%$ and changes in mental status in $17 \%-38 \%$. Seizures at stroke onset are more common in children than adults, affecting $15 \%$ $25 \%$, especially in those $<6$ years of age. The aim of clinical and imaging studies is to differentiate ischemic, hemorrhagic and non-vascular etiologies, with different stroke management cascades. ${ }^{9,19}$

The history and physical examination can provide information about the mechanism and etiology of stroke. A history of infection, head and neck trauma may result in dissection. A history of 
previous TIA and symptoms on the other side are important indicative of proximal embolism or bilateral arteriopathies (eg Moyamoya syndrome). Screening for family history of rapid onset of thrombotic events, cardiovascular disease and genetic disorders. Important elements of the physical findings include blood pressure, peripheral pulses, and auscultation for the presence of a neck bruit, ophthalmologic examination for the presence of arteriopathies and elevated intracranial pressure. The presence of skin lesions indicative of neurofibromatosis, Fabry disease or PHACES syndrome (posterior fossa abnormalities, hemangiomas, arterial cervicocerebral anomalies, cardiac defects, eye anomalies, sternal defects, and supraumbilical raphe). ${ }^{13}$

\section{STROKE SUPPORTING EXAMINATION IN CHILDREN}

The suitability of neuroimaging is important in establishing the diagnosis of ischemic stroke. CT scan of the head is performed to exclude intracranial haemorrhage but is weak for detecting acute brain ischemia, approximately 16-56\% in pediatric studies. Therefore, MRI was chosen as a neuroimaging investigation. Diffusion-weighted MRI (DWI) can detect brain ischemia early by showing hypointensity. Perfusion-weighted imaging shows abnormal perfusion of the brain. Conventional angiography is the gold standard for cerebrovascular imaging. Specific arteriopathies can be diagnosed, the presence of a string, double lumen or intimal flap sign on dissection, string of beads in vasculitis and arteriopathy in small caliber arteries. Conventional angiography should be performed in children with ischemic stroke of unknown specific etiology despite CT and MRA studies to characterize arteriopathies. Given that arteriopathy is the predominant etiology of both ischemic and hemorrhagic stroke, angiography is a step in the evaluation of children with acute stroke. ${ }^{8,16}$ Complete blood count, CRP, ANA, protein $\mathrm{C}$, protein $\mathrm{S}$, and echocardiography should be performed in all pediatric stroke populations. A patent foramen ovale is found up to 4 times as much in the pediatric stroke population. ${ }^{13}$

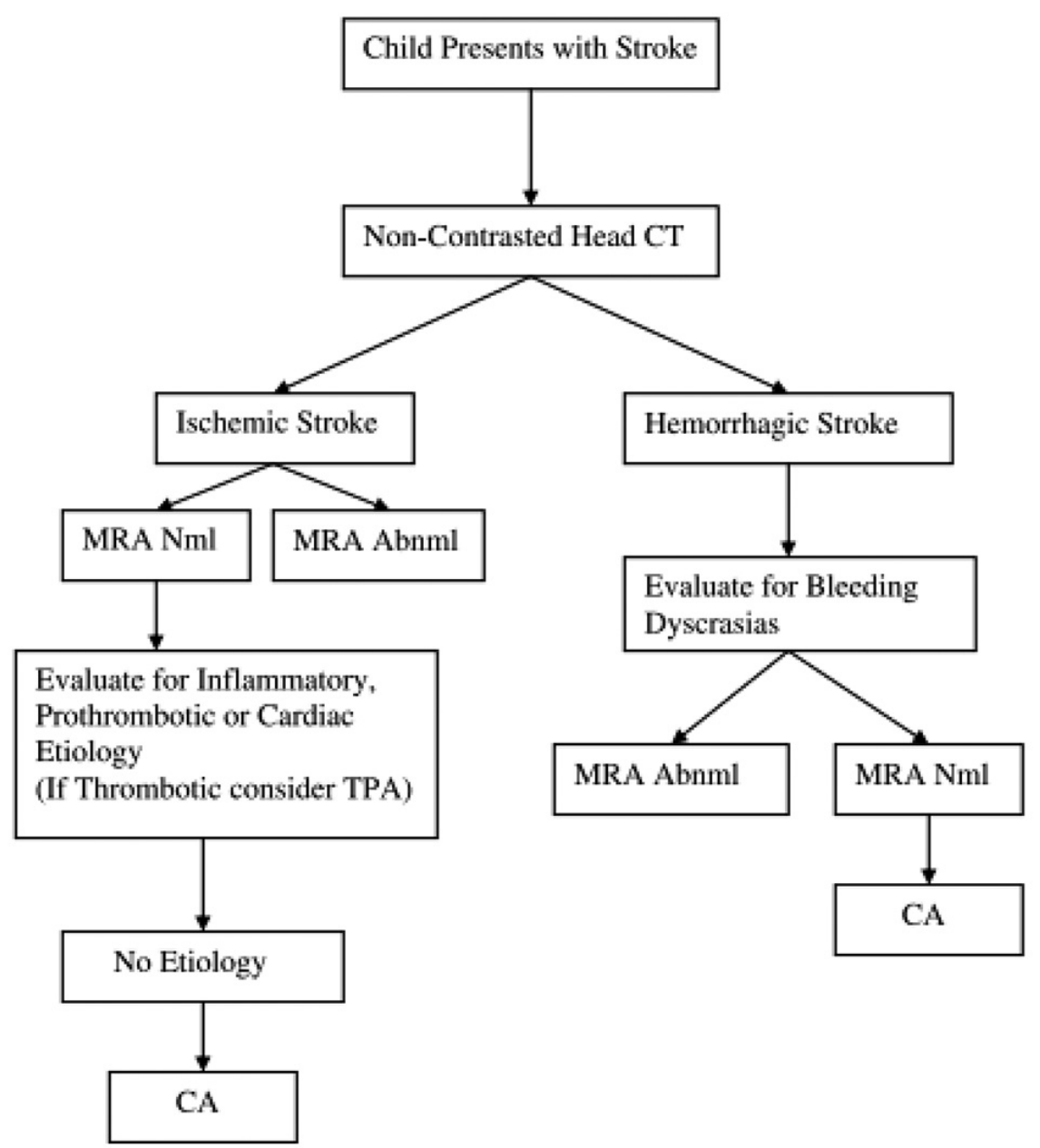

Figure 3. Flowchart for Early Evaluation of Stroke in Children. MRA or conventional brain angiography should be considered in children with stroke after bleeding dyscrasias and diathesis have been eliminated as the cause of the disease. ${ }^{16}$
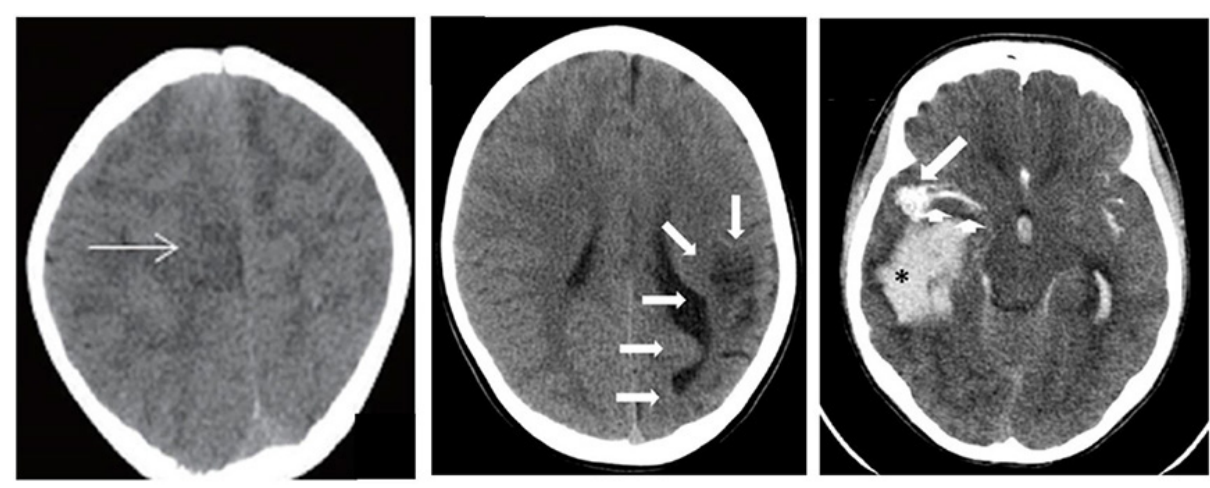

Figure 4. CT scan of the head in stroke. (a) Fibromuscular Dysplasia, a 14-month-old boy presented with 3 episodes of focal seizures of the left face, arm and leg without loss of consciousness and with residual left hemiplegia. Non-contrast CT shows a lesion in the right parasagittal cortex corresponding to an acute infarction (arrows) (Rajani et al., 2018) (b) A 6 year old female has seizures, physical examination shows signs of hyperreflexia and Babinski on the right, CT scan of the head shows arterial ischemic stroke. (c) A previously healthy 4-year-old boy then complained of headache and emesis. CT scan of the head with contrast axial section showing a right temporal with arteriovenous malformation (arrows) with branches of the middle cerebral artery (arrows), with intraparenchymal hemorrhage (asterisk). ${ }^{3,4}$ 


\section{STROKE THERAPY IN CHILDREN}

\section{Ischemic Stroke}

Accurate and prompt diagnosis is essential to enable neuroprotective management and intervention to co-annlize and prevent recurrence. Unfortunately, in some studies there has been a delay in diagnostic confirmation of more than 24 hours from onset. Rapid recognition of stroke and sensitivity of imaging contribute to delay in diagnosis. In ischemic stroke, irreversible ischemia of brain tissue occurs within minutes to hours after arterial occlusion. The time to irreversible tissue injury is shorter at the center of the infarct and around the penumbra area, the supply of collateral arteries can continue to perfuse the tissue. ${ }^{13}$

The neuroprotective strategy to balance the supply of metabolic substrates with the increased demand for tissue metabolism aims to increase the viability of brain tissue especially in the penumbra area and reduce the metabolic demand of neurons through control of fever and seizures. ${ }^{9}$ In contrast to the adult population, randomized clinical trials of stroke therapy were limited to children. Management is more general in nature to stabilize hemodynamic conditions, oxygen supplementation, and temperature control. Hypertension is associated with increased mortality and hypotension reduces penumbral tissue perfusion. Although the ideal blood pressure parameters for the treatment of pediatric acute stroke are unknown, it is considered to maintain blood pressure to $15 \%$ above the 90th percentile. Normalization of normal blood oxygen and carbon dioxide levels also improves cerebral perfusion and intracerebral autoregulation. Both hyperglycemia and hypoglycemia are associated with poorer neurologic outcome in stroke in critically ill adults and children but there are no specific data on pediatric stroke. Temperature control is very important; Various studies confirm that febrile conditions in acute ischemic stroke significantly increase mortality, disability, and length of hospitalization. The presence of seizures often occurs in AIS children, and can worsen ischemic conditions in the brain so that it must be monitored and given anticonvulsant therapy. ${ }^{13}$
Neuroprotective therapy is aimed at optimizing function, delivering oxygen and glucose to the penumbral tissue. CDP choline (citicoline) is a unique form of choline that readily crosses the blood brain barrier directly into the CDP choline tissue which has been shown to be useful in animal and human studies. Citicoline is approved as a therapy in Europe and Japan for use in stroke, head trauma and other neurological disorders. ${ }^{20}$ Citocoline reduces central nervous system injury by stabilizing cell membranes and reducing free radicals. Citicoline has been found to improve membrane integrity, increase acetylcholine formation, and contribute to the critical metabolic functions of nucleic acids (eg RNA and DNA) and protein synthesis. In the brain, in addition to increasing the synthesis of phospholipids, citicoline also inhibits the degradation of phospholipids. Citicoline shows long-term effects on attention-responsive cognition and behavior. Citicoline improves the function of blood vessels in the brain resulting in increased bioelectric activity, so it has cognitive benefits. Schematically, the effects of citicoline are an increase in blood vessels, an increase in immunity, an increase in bioelectricity, and an increase in cognitive. ${ }^{20}$

Alviarez and González reported the beneficial effects of citicoline administration in a double-blind study conducted in Venezuela. Leon-Jimenez et al. evaluated the correlation between citicoline exposure and functional outcome at discharge and at 30 and 90 days post-stroke, in a retrospective casecontrol design in a systematic descriptive database from three referral hospitals in Mexico. ${ }^{21}$ The clinical records of 173 consecutively enrolled patients were analysed, 86 of whom were treated with citicoline within the first 48 hours after acute ischemic stroke and the remaining 87 were untreated, randomly selected controls matched for age \pm 5 years and sex at hospital admission. Compared with controls, administration of citicoline for 30 days was associated with a lower risk of death $(\mathrm{p}=0.047)$ and with fewer complications in the hospital (mainly infection and sepsis, $\mathrm{p}=0.001$ ). In this observational study, citicoline use was associated with better functional status and lower short-term mortality rates. ${ }^{21}$

\section{THROMBOLYSIS AND THROMBECTOMY}

There are few findings regarding the benefits and safety of using tissue plasminogen activator (tPA) or thrombectomy in children. Thus, the pediatric stroke guidelines do not recommend this approach in children. Thrombolytic agents such as tPA convert plasminogen to plasmin to dissolve the thrombus. A meta-analysis in adults showed the effectiveness of tPA outweighs the risk when administered 4.5 hours after onset. However, the safety of thrombolysis in children is still poorly studied. ${ }^{9,13}$

\section{ANTITHROMBOTIC THERAPY}

Administration of antiplatelets and anticoagulants in the acute phase is not supported by evidence and expert recommendations. The first option is anticoagulant treatment with heparin, low molecular weight heparin (LMWH), or warfarin. Anticoagulants can theoretically be administered in certain circumstances such as cardiac embolism, doxical venous para-embolism, slow flow with severe stenosis, prothrombotic disorders, and dissection. The second option is antiplatelet treatment with aspirin, which may be more appropriate in arteriopathies where there is rapid thrombus formation. ${ }^{13}$

AHA recommendations (American Heart Association) recommend the use of low molecular weight heparin or acetylsalicylic acid ( $1 \mathrm{mg} / \mathrm{kg} /$ day) until the etiology of ischemic stroke in children is established. A prospective study showed that the effectiveness of low molecular weight heparin and acetylsalicylic acid was not significantly different in the prevention of secondary stroke. ${ }^{13}$

The most commonly used antiplatelet therapy in children with ischemic stroke is aspirin. There are guidelines that recommend antiplatelet agents as secondary prevention for noncardioembolic ischemic stroke. Aspirin is a primary antiplatelet agent for children with a good safety profile and inhibits platelet aggregation by reducing thromboxane A2 production. Other antiplatelet alternatives include clopidogrel, dipyramidol-release, ticlopidine, or glycoprotein 11 b-lll

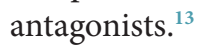


The 2008 AHA generally approved aspirin or LMWH for initial therapy in pediatric acute ischemic stroke. However, it is still unclear under what circumstances antiplatelet drugs or anticoagulants are the best initial and long-term secondary prevention in children. Decisions on antithrombotic treatment are difficult and individualization is required; Therefore, children with ischemic stroke should be referred and managed in a pediatric center with on-site expertise and protocols for pediatric stroke management. The availability of a hematologist's consultation with experience in stroke management is invaluable in managing children with thromboembolic stroke. For children with ischemic stroke anticoagulation or aspirin may be considered for the initial 5 to 7 days after presentation while the cause is being sought. Once the cause is known, further refinement of the medical approach can be applied. For stroke due to cardiac embolism (including neonates) or in individuals with previous thrombosis or known prothrombotic abnormalities, maintenance therapy usually consists of continued anticoagulation with LMWH or warfarin for 3 to 6 months or longer. In most children, follow-up therapy consists of aspirin at a dose of 3-5 mg/kg/day. The duration of aspirin therapy depends on the underlying condition and ongoing risk of recurrence. ${ }^{9}$ Most children are treated for 2 years to prevent most recurrent strokes from occurring. However, the duration of antithrombotic treatment in the setting of persistent arteriopathy is unknown. No clinical trials have evaluated whether antiplatelet drugs or anticoagulants are best; However, it is clear that the absence of antithrombotic therapy is associated with a 1.5- to 2-fold increased risk of recurrent stroke. ${ }^{9}$

In general, the 3 available guidelines recommend anticoagulation for secondary ischemic stroke prophylaxis in the presence of dissection, a cardioembolic source, or thrombophilia, with the duration of treatment depending on the condition. Administration of aspirin (3 to $5 \mathrm{mg} / \mathrm{kg}$ per day) may provide secondary stroke prevention. Aspirin is not given during influenza or varicella infection to minimize the risk of Reye's syndrome. The optimal duration of therapy is unknown. ${ }^{3,4}$
More than $50 \%$ of childhood stroke survivors have moderate or severe long-term neurological disorders and epilepsy. Rehabilitation should target physical, occupational, language, cognitive/behavioral, and psychosocial improvements. Compared to adults with stroke, children have better recovery potential; However, among children, a younger age at onset was associated with a worse long-term risk. Stroke in adults causes loss of function, whereas similar injuries in children cause loss of function and reduce the ability to acquire new things. Therefore, pediatric rehabilitation care must incorporate the patient's ageappropriate developmental knowledge and skills. Hemiparesis and motor disability are common sequelae of stroke. Hemidistonia can appear several months after a basal ganglia stroke. Rehabilitation programs that combine strength and aerobics, with assistive devices are generally recommended in children. ${ }^{13}$

\section{HEMORRHAGIC STROKE}

\section{Initial Management}

Stabilization of the patient and minimizing secondary brain injury are the goals of acute management. A neurosurgeon should be consulted immediately after the diagnosis of ICH or SAH. Basic management includes a neurologic examination to assess the severity of the injury, elevating the head to at least 30 degrees to facilitate venous drainage, administration of isotonic fluids, maintenance of normoglycemia and normothermia. Children with seizures should be treated with anticonvulsants. There are no data from clinical trials regarding medical therapy in children with ICH. Blood pressure was set with a target of the 95th percentile for age and sex. Elevated blood pressure in the setting of ICH may be a regulatory mechanism to maintain cerebral perfusion. Therefore, care must be taken to lower blood pressure in children with ICH so that secondary ischemia does not occur and monitoring of ICP should be considered. ${ }^{13}$

\section{Increased Intracranial Pressure}

Signs, Symptoms and Monitoring

Elevated ICP can occur as a direct mass effect of ICH or from intraventricular hemorrhage and hydrocephalus. This can occur in ICH or SAH. Monitoring for increased ICP should be considered in pediatric patients with $\mathrm{ICH}$ or $\mathrm{SAH}$ with decreased consciousness. An intraventricular catheter (IVC), also called extraventricular drainage (EVD), is often the monitoring tool of choice because it can measure ICP and regulate ICP through drainage of cerebrospinal fluid or intraventricular blood. ${ }^{13}$

Medical management to reduce acute ICP is usually temporary and includes PC02 hyperventilation of 25 to $30 \mathrm{~mm}$ $\mathrm{Hg}$, elevating the head at least 30 degrees to facilitate venous drainage, and using hyperosmolar therapy with hypertonic saline or mannitol. Corticosteroids have not been shown to be beneficial in adult studies. ${ }^{13}$

\section{Evacuation of intraparenchymal hemorrhage}

Treatment of a brain AVM depends on the size, pattern of venous drainage (superficial or deep) and location. In general, small, superficial sizes can be microresected whereas AVMs not included in this group require careful selection of therapy. Embolization (interventional radiological procedure) can facilitate microsurgery or radiosurgery by reducing the size of the AVM, but rarely completely eliminates the AVM. Radiosurgery exposure is not preferred in children because it can cause adverse cognitive effects. In this study of 38 children treated with radiosurgery for AVM with a follow-up of 42 months, $68 \%$ had an excellent outcome. ${ }^{22}$

Emergency supratentorial hematoma evacuation did not improve outcomes in adults with spontaneous ICH in surgical trials. The 2010 ICH guidelines for adult's state that patients with cerebellar hemorrhage, with decreased consciousness and brainstem compression, or with obstructive hydrocephalus from ventricular compression should undergo surgical evacuation of the hemorrhage. In children, lobar bleeding usually occurs, not internal bleeding. Hematoma evacuation may be necessary to reduce mass effect and to prevent or reduce herniation syndrome. ${ }^{13}$ 


\section{Hemicraniectomy}

Decompressive craniectomy can save life and function when sudden damage is seen in large artery ischemic stroke or ICH in an adult trial. In a study of 10 children with extensive MCA infarction, 7 underwent hemicraniectomies and all had good recovery. In the prospective cohort of children, 3 of 22 children with ICH underwent decompressive craniectomy; all are functionally independent. ${ }^{15}$

Surgical evacuation of hematomas for parenchymal hemorrhage deserves special mention because the management differs between adults and children. In pediatric patients with lobar hemorrhage with clinical deterioration due to the mass effect of bleeding, surgery has been shown to be beneficial in retrospective series studies. Children may require more urgent intervention to reduce ICP and prevent brain herniation. Furthermore, in adults, patients with cerebellar hemorrhage $>3 \mathrm{~cm}$ may benefit from early evacuation of the hematoma, because the site of bleeding often leads to tonsil herniation, hydrocephalus, or brainstem compression. ${ }^{22}$

\section{Seizures: Seizure Monitoring and Treatment}

Seizures are a common symptom and sequela of ICH and SAH. In this prospective cohort, more than $40 \%$ of children with ICH had seizures. Seizures can be treated with anti-seizure medications, at least in the acute period. Pediatric stroke guidelines from the American Heart Association recommend anticonvulsant prophylaxis in ischemic stroke and are not recommended in ICH. Continuous EEG monitoring should be considered in children who have l) decreased mental status or 2) movement or changes in vital signs suggestive of seizures that cannot be captured on routine EEGs. ${ }^{13}$

One of the promising therapies for hemorrhagic stroke in children, because it is minimally invasive and FDA approved for use in children, is recombinant factor VIIa. This drug facilitates hemostasis and is reserved for hemophiliac children who have systemic bleeding and who are resistant to factor VIII therapy. Factor VIIa is currently in phase III clinical trials in adult ICH; because up to $38 \%$ of patients with intraparenchymal hemorrhage will have expansion of the initial hemorrhage, stabilization of intracerebral hemorrhage can reduce bleeding volume and improve clinical outcome. Factor VIIa should ideally be within 4 hours of the onset of bleeding. ${ }^{22}$

\section{PROGNOSIS OF STROKE IN CHILDREN}

\section{Ischemic Stroke}

Stroke mortality in children is $5-10 \%$ and is in the top 10 causes of death in children. ${ }^{8}$ Brain injury from repeated stroke is a major concern in infants and children. Fullerton et al evaluated the risk of recurrent ischemic stroke from an international perspective. They found the cumulative rate of stroke recurrence was $6.8 \%$ (95\% CI.4.6\%-10\%) after 1 month and $12 \%$ (95\% CI, $8.5 \%-15 \%)$ after 1 year in most children. undergoing antithrombotic treatment. The predictor of stroke recurrence was the presence of arteriopathy, which resulted in a 5 -fold increased risk of recurrence. This risk occurs despite the increased use of antithrombotic agents. ${ }^{14}$

Although previous studies have shown a mortality rate of $20 \%$ after stroke in children, more recent studies have found that mortality is quite a bit lower. A recent population-based retrospective cohort from the United States that included 123 children with ischemic stroke from a population base of 2.3 million children found that the overall mortality at hospital discharge was $4 \%$. Similarly for Pediatric Stroke in Canada, stroke-specific mortality was $5 \%$ above the median at 2.9 years of follow-up. Outcome after hospital discharge was 484 of 701 (69\%) children with ischemic stroke. Normal neurological outcome is documented in $30 \%$. Although $70 \%$ had neurological deficits, $36 \%$ had mild, $23 \%$ moderate, and $10 \%$ had severe deficits. Mallick et al found that of 94 British children with ischemic stroke, $10 \%$ died. One year after stroke, 50\% of the 78 survivors had a Child Stroke Outcome Measurement Score <1. Child Stroke Outcome Measurement Score 1 indicates moderate functional impairment in 1 domain (motor, language, cognition, or behavior), so if Child Stroke Outcome Score $<1$ is very good; mild until no disturbance occurs. In 95 Swiss children with ischemic stroke, long-term outcome was assessed at 6.9 years of followup. Telephone interviews were used to obtain modified Rankin Scale scores. At this point in time, $14 \%$ had died. As has been reported in other studies, about half died from underlying systemic disease compared to stroke itself. Normal results were found in $27 \%$ of children and $28 \%$ had mild impairment, with a modified Rankin scale of $1.98 .^{9}$

\section{Hemorrhagic Stroke}

In a population-based cohort of 116 children with ICH, 11 children (9.5\%) had recurrent ICH at a median of 3.1 months from initial bleeding. Nine out of 11 recurrences occurred in children with vascular disorders, 6 of whom were untreated. Two children with a medical etiology of recurrent ICH within 1 week of bleeding events. ${ }^{13}$ Estimates of mortality after childhood range from 5\% - 54\%. Predictors of death in one study included Hispanic ethnicity, older age (11 to 18 years vs I to 10 years), coagulopathy, and coma. Worse outcomes were related to ICH volume, change in mental status within 6 hours of onset, infratentorial location, GCS less than or equal to 7 at admission, cause of bleeding aneurysm, age younger than 3 years at time of ICH, and underlying haematological disorders. In one prospect cohort of 72 children and neonates with $\mathrm{ICH}$, the 1- and 2-year seizure-free survival rates were $82 \%$ and $67 \%$, respectively. ${ }^{13}$

\section{CONCLUSION}

Stroke in children was defined as the clinical and radiological manifestation of stroke, with radiological evidence of cerebral infarction or hemorrhage. The etiology is very different, and diagnostic procedures are often more in-depth to look for etiology. The treatment dependent on the etiology and children condition. Early recognition of childhood stroke is very important with the implementation of targeted and neuroprotective therapies.

\section{CONFLICT OF INTEREST}

The authors report no declarations of interest. 


\section{ETHICS CONSIDERATION}

This article has followed the COPE and ICMJE protocol of publication ethics prior to the study being conducted

\section{FUNDING}

None.

\section{AUTHOR CONTRIBUTIONS}

All authors equally contribute to the study from the conceptual framework, data acquisition, data analysis, until reporting the study results through publication.

\section{REFERENCES}

1. Golomb MR, Fullerton HJ, Nowak-Gottl U, Deveber G; International Pediatric Stroke Study Group. Male predominance in childhood ischemic stroke: findings from the international pediatric stroke study. Stroke. 2009;40(1):52-57.

2. Rajani NK, Pearce K, Campion T, Salpietro V, Planells M, Chong W, et al. Pediatric stroke: current diagnostic and management challenges. Quant Imaging Med Surg. 2018;8(10):984-991.

3. Bernson-Leung ME, Rivkin MJ. Stroke in Neonates and Children. Pediatr Rev. 2016;37(11):463-477.

4. Zimmer JA, Garg BP, Williams LS, Golomb MR. Age-related variation in presenting signs of childhood arterial ischemic stroke. Pediatr Neurol. 2007;37(3):171-175.

5. DeVeber G, Kirkham F. Guidelines for the treatment and prevention of stroke in children. Lancet Neurol. 2008;7(11):983-985.

6. Harnsberger HR, Osborn AG, Ross JS, Moore KR, Salzman KL, Carrasco CR, et-al. Diagnostic and Surgical Imaging Anatomy. Lippincott Williams \& Wilkins. 2006. [Available at: https:// radiopaedia.org/articles/circle-of-willis]

7. Hall JE, Hall ME. Guyton and Hall textbook of medical physiology e-Book. Elsevier: Health Sciences. 2020.

8. Tini K, Samatra IDPGP, Wiryadana KA, Supadmanaba IGP. Clinical profile of patients with cerebrovascular disease at Stroke Unit, Sanglah General Hospital, Denpasar, Bali. Bali Medical Journal. 2020;9(1):129-136.

9. Ferriero DM, Fullerton HJ, Bernard TJ, Billinghurst L, Daniels SR, DeBaun MR, et al. Management of Stroke in Neonates and Children: A Scientific Statement From the American Heart Association/American Stroke Association. Stroke. 2019;50(3):e51-e96.

10. Lehman LL, Khoury JC, Taylor JM, Yeramaneni S, Sucharew H, Alwell K, et al. Pediatric Stroke Rates Over 17 Years: Report From a PopulationBased Study. J Child Neurol. 2018;33(7):463467.

11. Gerstl L, Weinberger R, von Kries R, Heinen F, Schroeder AS, Bonfert MV, et al. Risk factors in childhood arterial ischaemic stroke: Findings from a population-based study in Germany. Eur J Paediatr Neurol. 2018;22(3):380-386.

12. Venkatesan C, Wainwright MS. Pediatric endocarditis and stroke: a single-center retrospective review of seven cases. Pediatr Neurol. 2008;38(4):243-247.

13. Swaiman KF, Ashwal S, Ferriero DM, Schor NF, Finkel RS, Gropman AL, et al. Pediatric Neurology: Principles and Practice. Sixth Edition: Elsevier. 2018.

14. Fullerton HJ, Wintermark M, Hills NK, Dowling MM, Tan M, Rafay MF, et al. Risk of Recurrent Arterial Ischemic Stroke in Childhood: A Prospective International Study. Stroke. 2016;47(1):53-9.

15. Beslow LA, Jordan LC. Pediatric stroke: the importance of cerebral arteriopathy and vascular malformations. Childs Nerv Syst. 2010;26(10):1263-1273

16. Gumer LB, Del Vecchio M, Aronoff S. Strokes in children: a systematic review. Pediatr Emerg Care. 2014;30(9):660-664.

17. Abend NS, Beslow LA, Smith SE, Kessler SK, Vossough A, Mason S, et al. Seizures as a presenting symptom of acute arterial ischemic stroke in childhood. J Pediatr. 2011;159(3):47983.

18. Scothorn DJ, Price C, Schwartz D, Terrill C, Buchanan GR, Shurney W, et al. Risk of recurrent stroke in children with sickle cell disease receiving blood transfusion therapy for at least five years after initial stroke. J Pediatr. 2002;140(3):348-54.

19. Tsze DS, Valente JH. Pediatric stroke: a review. Emerg Med Int. 2011;2011:734506.

20. Warsiki E. CDP choline (Citicoline= Nicholin) therapy on some cases of children with organic brain syndrome. Folia Medica Indonesiana, 2004;40(1):43-47.

21. Secades JJ. Citicoline: pharmacological and clinical review, 2016 update.. Rev Neurol. 2016;63(S03):S1-S73.

22. Jordan LC, Hillis AE. Hemorrhagic stroke in children. Pediatr Neurol. 2007;36(2):73-80.

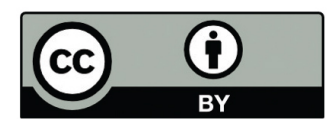

This work is licensed under a Creative Commons Attribution 